Part 5

Smart Coatings 



\section{Comparison between the effect of iron oxide and micaceous iron oxide into intermediate layer on zinc rich epoxy primer by EIS}
B. F. de Castro'
M. I. Valentini ${ }^{1}$
R. Macanhan ${ }^{2}$
M. A. C. Berton'

\section{Abstract}

Protective epoxy organic coatings can be modified in their formulations to reach better characteristics and protective properties against corrosion. In order to study and evaluate the changes in the organic coating regarding its protection capacity against corrosion, the electrochemical impedance spectroscopy (EIS) employing a $3.5 \% \mathrm{NaCl}$ solution (in mass), was used. Carbon steel samples were covered with the organic coating (primer + intermediate layer). For comparison purposes, the same zinc rich epoxy primer was used in analyzed samples. The intermediate layer with and without micáceo iron oxide was applied over the primer. The results obtained by EIS showed significant good results for samples with micaceo iron oxide into the intermediate layer. The EIS measurements confirm that coating containing MIO present a higher value of resistivity comparing with coating without micaceo iron oxide as pigment. MIO coating presented high resistivity comparing the coating with spherical iron oxide pigment due the longer diffusion pathways produced by micáceo iron oxide lamellar shape.

Keywords: paint, primer rich zinc, micáceo iron oxide, electrochemical impedance spectroscopy, corrosion.

\footnotetext{
1 SENAI Institute of innovation in Electrochemistry, Curitiba - Paraná - Brazil.

2 Renner Hermann S.A. Curitiba - Paraná - Brazil..
} 


\section{Introduction}

An industrial paint system is composed of three layers namely: a primer layer, an intermediate layer and a topcoat layer. The primer layer are those that are applied directly to the substrate, therefore, is the paint responsible for the adhesion of the coating system to the substrate to protect and are those containing the composition said anticorrosive pigments. The intermediate paint is typically used in paint systems with the function of increasing coating thickness, with the aim of increasing the barrier protection thereof. The finishing paints (topcoat) have the function to check the chemical resistance coating. It is in direct contact with corrosive media, have in most cases good resistance to ultraviolet rays and are the paint that give the finish color of the coatings painting. For each kind of application, there is a specific composition for each layer of the coating system. The total coating system provide a barrier against corrosive species present in the environment, due to their high resistance to oxygen, water and ions transport. The effectiveness of the corrosion protection provided by the coating requires good adhesion properties between the metallic substrates and the coating, coating stability for long periods, and low permeability towards corrosive species. Longer and more effective corrosion protection is achieved by adding corrosion inhibitors and pigments [1-4]. The corrosion inhibition depends on the nature of the pigment and its distribution in the polymeric coating. In the case of intermediate layer, each mineral filler reflects on each of the properties of a paint, establishing a correlation between the accepted parameter fillers in the formulations and the technological performance of the paint.

Some intermediate layer like mica is add into the formulation to improve the barrier effect. Recently, layer to improve the barrier effect hindering the penetration of corrosive electrolyte to the protective film it was added micaceous iron oxide in a proportion of $80 \%$ of the dry film. The micaceous iron oxide term refers to its resemblance particle structure with mica [5-6], similar to what is on the lamellar form of the particles, which make it difficult to pass fluids by layer increasing the way to go another important property that this oxide can aggregate and increased roughness, which can contributes significantly to the mechanical adhesion between the layers [6]. Micaceous iron oxide known also as specular hematite and flaky hematite is a natural mineral ore that consist after refining mainly of $\mathrm{Fe}_{2} \mathrm{O}_{3}$. Micaceous iron oxide differs in the form and shape from the well-known iron oxides that are used as colour pigments. MIO shows a flaky, lamellar structure, the colour is dark grey with a metallic sheen. MIO is an inert pigment without electrochemical activity [7].

The contribution of this study is use the electrochemical impedance spectroscopy technique (EIS) for assessing of the effect of the new formulation of 
intermediate layer containing micaceous iron oxide as a substitute of spherical iron oxide, over the same primer layer, a zinc rich epoxy, on the resistivity of the coating in the corrosion protection. The EIS is one of the most used electrochemical techniques for determination of the efficiency of organic coating against corrosion $[8,9]$. In the segment of industrial painting, there is the need for a more precise determination regarding the coating guarantee. This is important because it allows a better control of the protective system efficiency.

\section{Experimental}

It was made in the metallic substrate an abrasive blasting to obtain the surface roughness equivalent to the Sa $2 \frac{1}{2}$ level. The organic coating was applied to the substrate by an airless spray in the following order: first it was applied to the primer zinc rich epoxy followed by the intermediate layer after drying of the first layer. The resulting thickness of the dry layer was between $100 \mu \mathrm{m}$ and $120 \mu \mathrm{m}$. Two types of intermediate were used, i.e, an intermediate containing micaceous iron oxide additive $(\mathrm{MIO})$ as a substitute of mica and the same intermediate without MIO.

The EIS analysis was carried out in a potentiostat/galvanostat (VersaStat 3 model from AMETEK) with a frequency range between $1.0 \mathrm{MHz}$ and $1.0 \mathrm{mHz}$ using the $\mathrm{Ag} / \mathrm{AgCl}$ as reference electrode and a platinum counter electrode with an area significantly higher than that one as working electrode. The electrolyte was a $3.5 \%$ of $\mathrm{NaCl}$ aqueous solution. The working electrode was a carbon steel with organic coating with an active area of $15 \mathrm{~cm}^{2}$. The measuring cell was built by placing a plastic cup with $100 \mathrm{~cm}^{3}$ volume over the organic coating and sealed with an epoxy resin. Figure 1 shows the scheme for the EIS experimental analysis. All EIS measurements were carried out into a Faraday Cage.

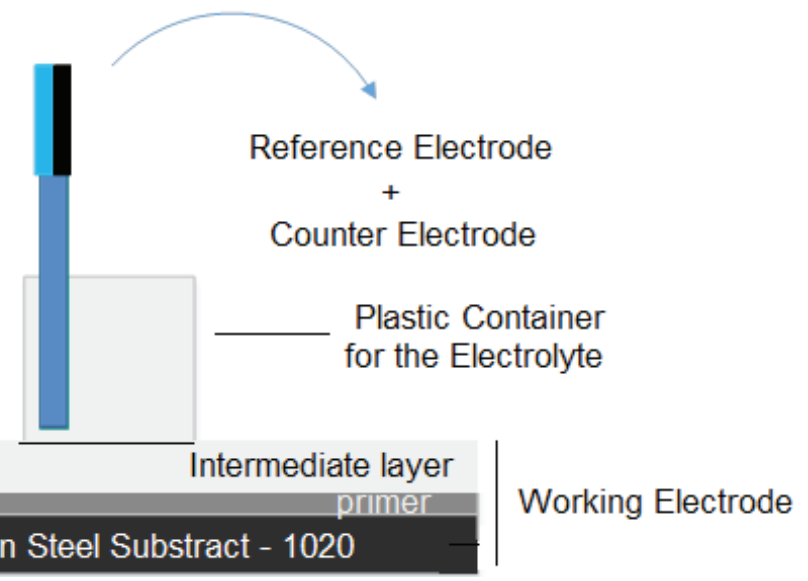

Figure 1 Schematic drawing of the three electrode electrochemical cell. 


\section{Results e discussion}

The electrochemical impedance tests were carried out for the samples left at their open circuit potential in the $\mathrm{NaCl} 3.5 \%$ electrolyte. AC impedance data were obtained by applying a sinusoidal wave perturbation at frequencies between $1.0 \mathrm{MHz}$ and $1.0 \mathrm{mHz}$. The amplitude of the AC voltage signal was $10 \mathrm{mV}$. The representation in Figure 2 is known as Nyquist plot where the ordinate axis represents the imaginary component of impedance and the $\mathrm{x}$-axis is the real component of the impedance. In the figure, high frequencies are located in the region of low impedance and the low frequencies in the high impedance region. The Figure 2 shows the EIS results for samples covered with primer zinc rich epoxy and with intermediate layer containing iron oxide as pigment. Samples with micaceous iron oxide (MIO) as substitute of iron oxide in the intermediate layer is presented in Figure 3 Both Figures show the results obtained for times between $24 \mathrm{~h}$ and 600 $\mathrm{h}$ of immersion of coating in $3.5 \% \mathrm{NaCl}$ aqueous solution.

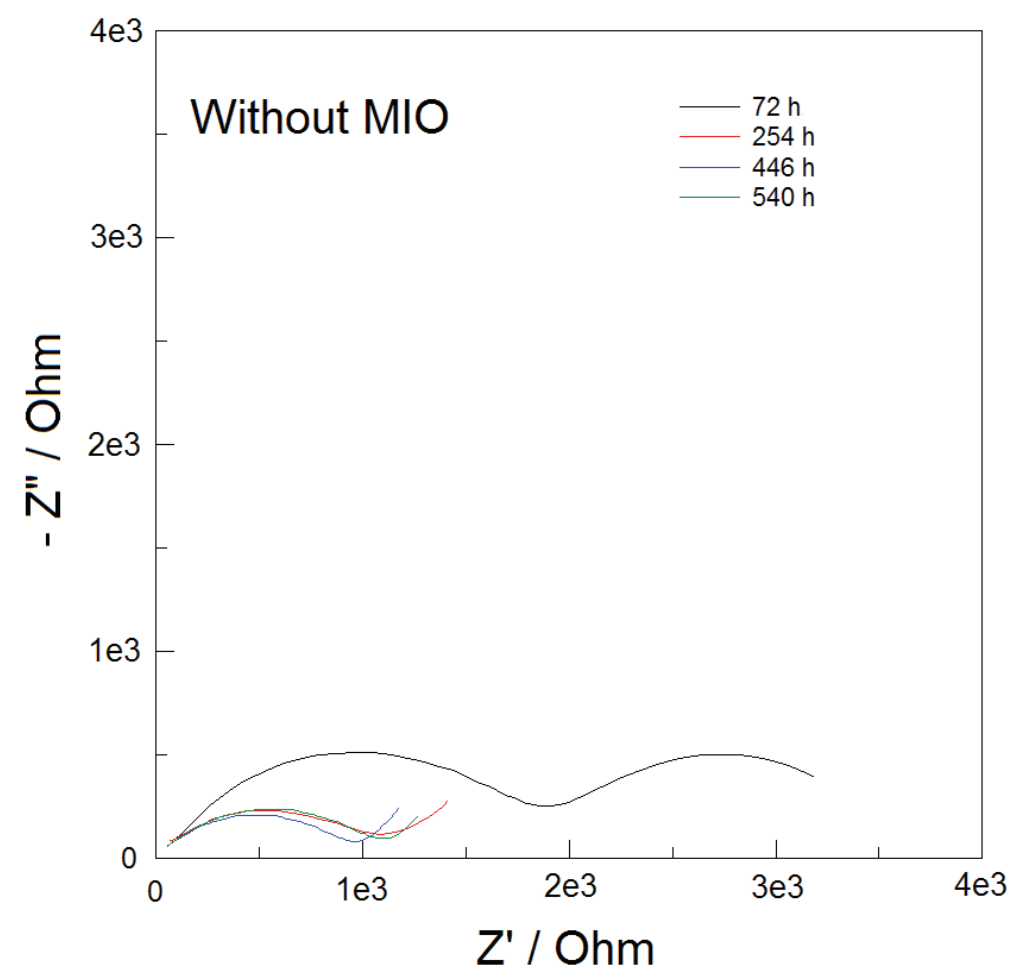

Figure 2 Evolution of Nyquist diagrams as a function of immersion time in 3.5\% NaCl aqueous solution for sample with intermediate layer containing iron oxide as pigment. Primer layer is a zinc rich epoxy paint. Total thickness of coating $120 \mu \mathrm{m}$. 


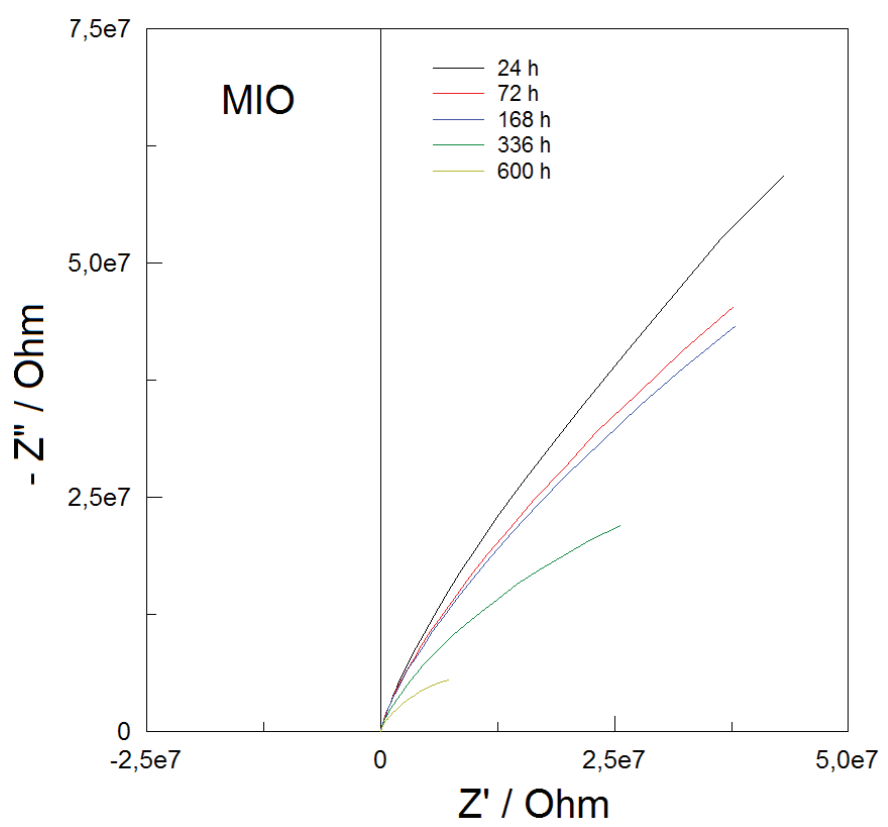

Figure 3 Evolution of Nyquist diagrams as a function of immersion time in 3.5\% $\mathrm{NaCl}$ aqueous solution for sample with intermediate layer containing micaceo iron oxide ( $80 \%$ solids in dry film of paint). Primer layer is a zinc rich epoxy paint. Total thickness of coating $120 \mu \mathrm{m}$.

To assess the level of protection against corrosion in $\mathrm{NaCl} 3.5 \%$ solution, i.e., resistance values of the organic coating, with and without micaceous iron oxide, were obtained using an equivalent circuit model shown in Figure 4. In the Figure, $\mathrm{R} 1$ is the resistance of the solution, $\mathrm{R} 2$ is the resistance of the organic coating and CPE1 is the constant phase element of the coating (primer + intermediate layer). $\mathrm{R} 3$ is considered to be the charge transfer resistance at metal-paint interface and CPE2 the constant phase element (the double layer capacitance). The equivalent circuit is similar that described in the literature [10-12].

Changes in the magnitudes of the resistivity of the coating as a function of the immersion time in the test solution, $3.5 \% \mathrm{NaCl}$, are plotted in Figure 5. It can be observed the resistivity of coating containing MIO present a higher resistivity comparing with coating without micaceous iron oxide as pigment. The lamellar shape of this particular form of iron oxide offers greatly enhanced barrier protection. The increase in corrosion resistance of the coating reinforced with MIO particles is considerably greater than the one reinforced with only iron oxide. This result may be attributed to the different mechanisms of permeation of aggressive species into the coating layers. 
The resistivity of coating with MIO is increased by the reduction of the transport of aggressive species into the polymer matrix by producing longer diffusion pathways due to tortuosity produced by micaceous iron oxide [3] Figure 6 shows an illustrative picture comparing a metal sample covered by paint with iron oxide particles and another with micaceous iron oxide.

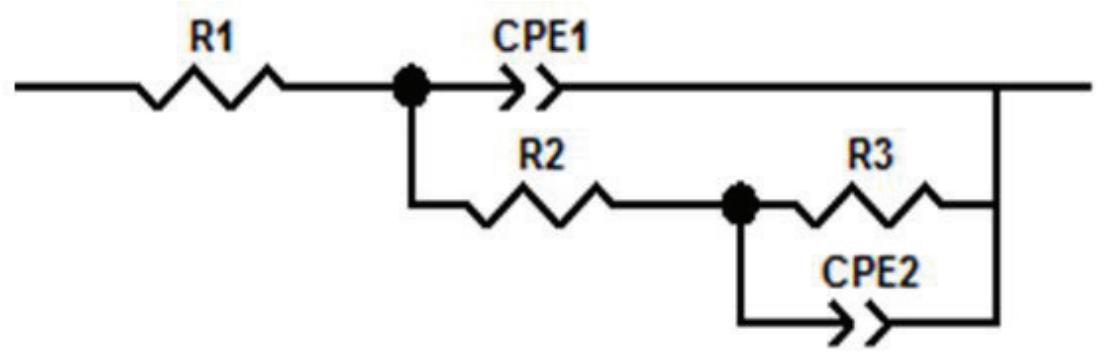

Figure 4 Electrical circuits used to simulate the ElS results of the carbon steel covered by zinc rich epoxy primer and an intermediated layer.

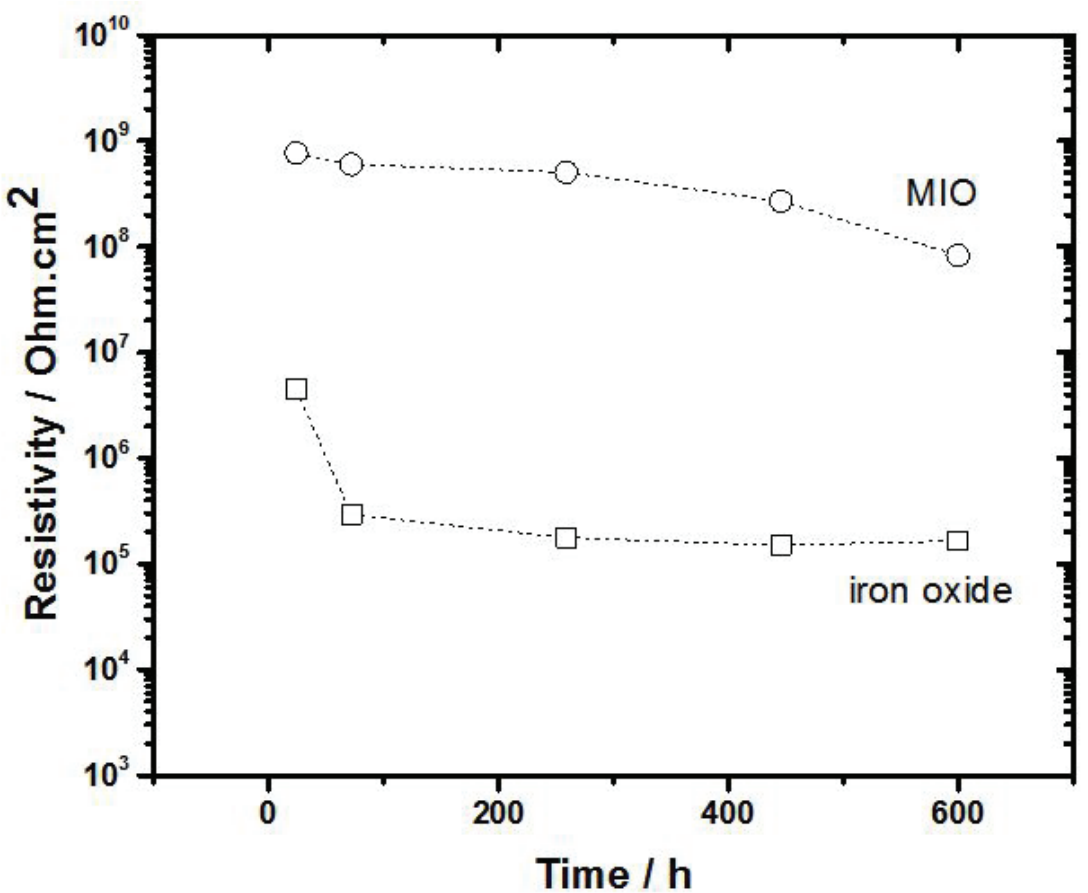

Figure 5 Changes of the resistivity of coating (primer + intermediated layer) as a function of immersion time in $3.5 \%$ $\mathrm{NaCl}$ aqueous solution. MIO: coating with intermediate layer containing micaceous iron oxide. Iron 0xide: intermediate layer iron oxide particles as pigments. 
Generally, pigments used in the coatings industry are roughly spherical in shape and may contribute to improve some properties like opacity, colour, abrasion-resistance, or mere as fillers. On the other hands, lamellar pigments, such as micaceous iron oxides, are used specifically for their ability to greatly enhance the coating's barrier properties [7]. The coating containing MIO in its intermediate layer gave higher values in resistivity showing increased corrosion protection due the increase barrier effect. This result was attributed due the property of their lamellar structure that has similar properties of Mica, which hinders the penetration of the electrolyte through the intermediate barrier film causing an increased protective effect.

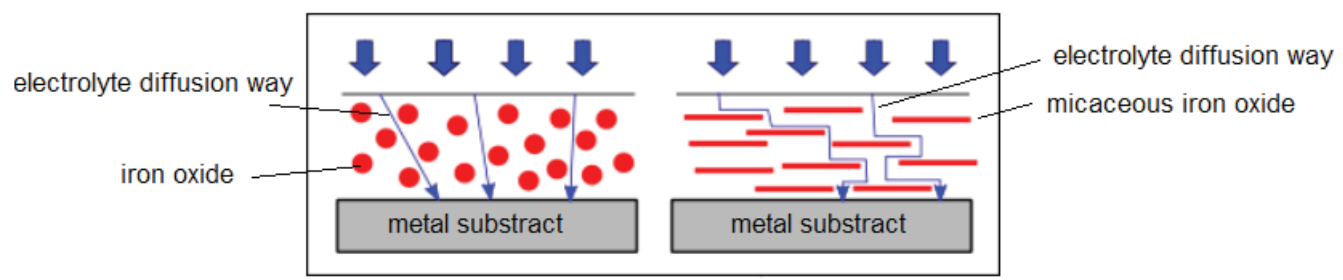

Figure 6 Schematic comparison between a paint system with iron oxide and other paint system with micaceous iron oxide. The picture of the righ side allows observing a significant increase in the barrier effect due the longer electrolyte diffusion way to reach the metal substrate from surface.

\section{Conclusions}

The results obtained by EIS showed significant good results for samples with micaceo iron oxide into the intermediate layer. The EIS measurements confirm that coating containing MIO present a higher value of resistivity comparing with coating without micaceous iron oxide as pigment. MIO coatings offer excellent barrier protection on corrosion comparing with the coating containing iron oxide spherical particle.

MIO coating presented high resistivity comparing the coating with spherical iron oxide pigment due the longer diffusion pathways produced by micaceous iron oxide lamellar shape.

\section{References}

[1] M. Kendig, D.J. Mills, An historical perspective on the corrosion protection by paints, Prog. Org. Coat. (2016), http://dx.doi.org/10.1016/j.porgcoat.2016.04.044 
[2] L. W. McKeen, Pigments, Fillers, and Extenders. Fluorinated Coatings and Finishes Handbook (Second Edition), 2016, Pages 83-106.

[3] B. N. Popov. Corrosion Inhibitors. Corrosion Engineering, 2015, Pages 581-597.

[4] J. Cruz, R. Martınez, J. Genesca, E. Garcia-Ochoa. Experimental and theoretical study of 1-(2-ethylamino)-2-methylimidazoline as an inhibitor of carbon steel corrosion in acid media. J. of Electroanalytical Chemistry 566 (2004) 111-121.

[5] S.Y. Arman, B. Ramezanzadeh, S. Farghadani, M. Mehdipour, A. Rajabi. Application of the electrochemical noise to investigate the corrosion resistance of an epoxy zinc-rich coating loaded with lamellar aluminum and micaceous iron oxide particles. Corrosion Science 77 (2013) 118-127.

[6] P. Kalenda, A. Kalendová, V. Štengl, P. Antoš, J. Šubrt, Z. Kváca, S. Bakardjieva. Properties of surface-treated mica in anticorrosive coatings. Progress in Organic Coatings 49 (2004) 137-145.

[7] M. Mahdavian, M.M. Attar, Another approach in analysis of paint coatings with EIS measurement: Phase angle at high frequencies. Corrosion Science 48 (2006) 4152-4157.

[8] F. Mansfeld, M. W. Kendig, S. Tsai, Evaluation of Corrosion Behavior of Coated Metals with AC Impedance Measurements, CORROSION. 1982;38(9):478-485.

[9] John M. McIntyre, Ha Q. Pham. Electrochemical impedance spectroscopy; a coatings optimizations. Progress in Organic Coatings 27 (1996) 201-207.

[10] P. L. Bonora, F. Deflorian, L. Fedrizzi. Electrochemical impedance spectroscopy as a tool for investigation under paint corrosion. Ekcrrochimro Acta. Vol. 41. Nos. 1/8. pp. 1073. 108.2, 1996.

[11] B. Nikravesh, B. Ramezanzadeh, A.A. Sarabi, S.M. Kasiriha. Evaluation of the corrosion resistance of an epoxy-polyamide coating containing different ratios of micaceous iron oxide/Al pigments. Corrosion Science 53 (2011) 1592-1603. 
[12] C.M. Abreu, M. Izquierw, M. Keddam, X.R. NVOA and H. Takenouti. Electrochemical behavior of zinc rich epoxy paints in $3 \% \mathrm{NaCl}$ Solution. Electrochimica ACM. Vol. 41. No. 15. pp. 240-2415. 1996. 
